\title{
Electrical field induced growth of triangular nanometer structures on $\mathrm{WSe}_{2}$
}

\author{
J. Boneberg, M. Lohrmann, M. Böhmisch, F. Burmeister, M. Lux-Steiner, P. Leiderer \\ Fakultät für Physik, Universität Konstanz, D-78434 Konstanz, Germany \\ Received: 4 April 1995
}

\begin{abstract}
We report on the conditions for the growth of triangular structures on $\mathrm{WSe}_{2}$ surfaces in scanning tunneling experiments with a vertical dimension of one layer (Se-W-Se) and up to $200 \mathrm{~nm}$ in horizontal direction. Experiments carried out in different atmospheres (ambient air, dry $\mathrm{N}_{2}$, dry $\mathrm{O}_{2}$ ) suggest that the growth is directly related to the presence of a thin physisorbed water layer on the surface of $\mathrm{WSe}_{2}$. Furthermore examinations under different scanning and bias conditions show that the electric field of the tip induces the growth of these nanometer structures.
\end{abstract}

PACS: $81.60 . c p ; 82.30 .-\mathrm{b} ; 85.42 .+\mathrm{m}$

The use of a scanning tunneling microscope to modify surfaces on a nanometer scale has been demonstrated in many experiments [1]. Some of them have shown that it is possible to "etch" structures with nanometer dimensions in lateral direction and a depth of a single molecular layer [2-5]. Whereas this etching process has been shown for different metal chalcogenides [2,3], the details of the process are not clear at all. Suggested mechanism are for example 1) the production of reactive species from organic impurities on the surface in the presence of the electric field and/or the current of the tunneling tip, 2) a thermal effect, 3) field assisted evaporation and 4) abrasion via direct sample tip contact. Some of these materials (but not the $\mathrm{WSe}_{2}$ examined here) can be etched with the atomic force microscope (AFM) as well [4]. The application of this etching process towards a production of individual structures on a nanometer scale so far is hampered by the fact that scanning over the structures leads to further growth and therefore to a change of the structures $[2,5]$.

Motivated by the previous results [2-5] we have studied in detail the conditions for the etching of such structures with the STM on $\mathrm{WSe}_{2}$. Our measurements demonstrate that the etching process is controlled via the electric field at the surface of the semiconductor.
The $\mathrm{WSe}_{2}$ samples have been grown using the vapor phase transport method [6]. They are positively doped with a dopant concentration of $p=10^{16} \mathrm{~cm}^{-3}$. The first part of experiments was performed in air using a standard STM set-up, the second part in a HV chamber with a base pressure of $3 \cdot 10^{-6}$ mbar. The tunneling tips (Pt-Ir) were cut mechanically. Other tip materials $(\mathrm{Au}, \mathrm{W})$ have been used as well with similar results. All pictures were taken in the constant current mode, at a rate of usually one picture in 1-2 min. During the data storage the tip was positioned in the center of the scanned area under normal tunneling conditions.

For the generation of an individual structure an initially atomically flat area of the surface was chosen, and the scanning of the tip was stopped at the central position. Then during tunneling at positive sample bias of $+1 \mathrm{~V}$ and a current of $0.5 \mathrm{nA}$ a single additional voltage pulse of $+5 \mathrm{~V}$ was directly superimposed on the tunneling voltage. The pulse length was gradually increased from $10 \mathrm{~ns}$ to up to $500 \mathrm{~ns}$ until a depression with a diameter of about $2 \mathrm{~nm}$ could be found in the picture taken after the pulse. This depression grew towards equilateral triangular structures during the subsequent scans. The evolution of the $\mathrm{WSe}_{2}$ surface after the application of such an electrical pulse is illustrated in Fig. 1, and is similar to the observation reported by Akari et al. [3].

\section{Influence of the scanning and the bias conditions on the growth}

This set of experiments, carried out in ambient air, was started with a measurement where the tip was scanned across the surface without any major interruptions. Figure 2 shows the length of one edge of a triangle as function of time for a typical run. It is found that the edge length increases linearly, as reported in the preceeding paper [5], corresponding to a constant growth velocity $\mathrm{v}_{\mathrm{q}}$, $7.5 \cdot 10^{-2} \mathrm{~nm} / \mathrm{s}$ in this case. It turned out that $\mathrm{v}_{\mathrm{q}}$ varies considerably from day to day, as already observed in other experiments [2,5], with values between 0.01 and $1 \mathrm{~nm} / \mathrm{s}$ in our experiments. 


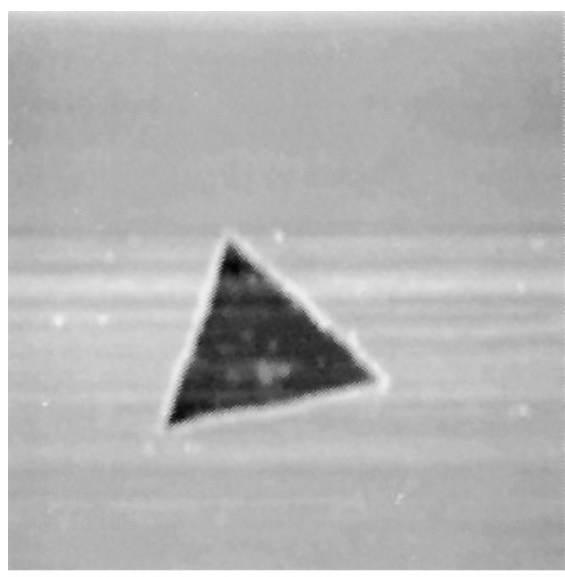

$514 \mathrm{~s}$

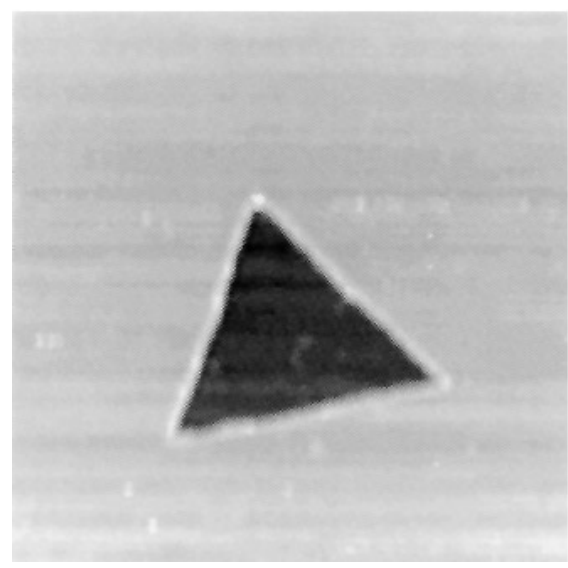

$666 \mathrm{~s}$

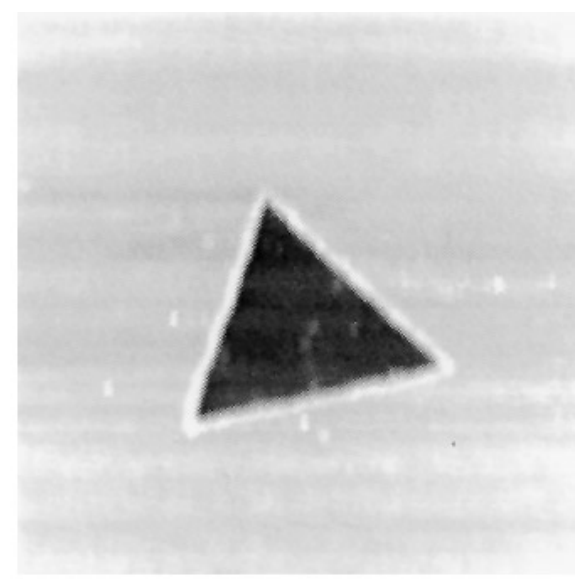

$620 \mathrm{~s}$

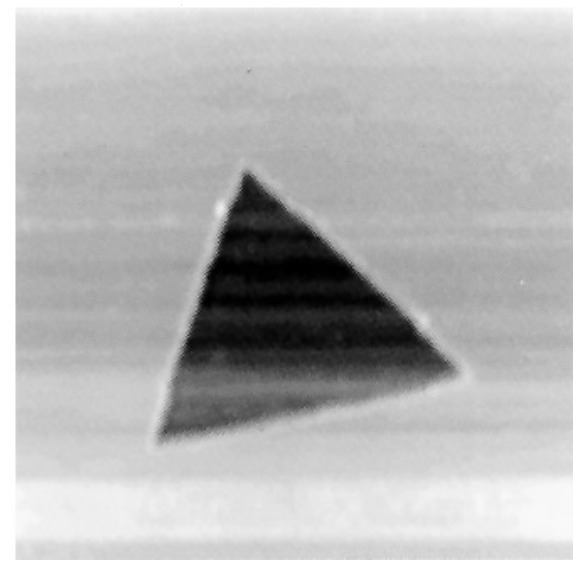

$766 \mathrm{~s}$

Fig. 1. Topography of the evolution of a triangular structure on $\mathrm{WSe}_{2}$ during scanning at a bias of $+1 \mathrm{~V}$ and a tunneling current of $1 \mathrm{nA}$. The pictures shown here $(200 \mathrm{~nm} * 200 \mathrm{~nm})$ were taken at $514,620,666,766 \mathrm{~s}$, respectively, after the structure had been nucleated

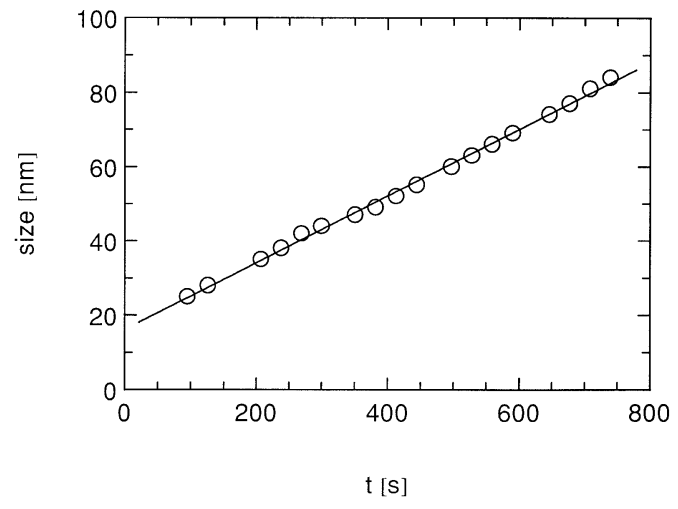

Fig. 2. Size (i.e. length of the side) of a triangular structure, such as in Fig. 1, as function of time (the values on the abscissa indicate the moments of data storage). Open circles: experimental data; solid line: linear fit
Figure 3 shows the evolution of another triangle, but now the scanning was interrupted several times: for $700 \mathrm{~s}$, as the triangle had reached a size of about $40 \mathrm{~nm}$ (interval I), for $600 \mathrm{~s}$ at a size of $60 \mathrm{~nm}$ (interval II), and for $2500 \mathrm{~s}$ at a size of $80 \mathrm{~nm}$ (interval III). During the scanning pauses of the intervals I and II the tip was positioned in the center of the triangle under normal tunneling conditions. As the interpolation shows the triangle continues to grow at constant rate, hence the growth process seems to be independent of the scanning. Completely different, however, is the behavior during interval III: there the tip was retracted by $300 \mathrm{~nm}$ in z-direction, and quite obviously no further growth occurred during this period.

A similar experiment, yet with an at first glance contradictory result, is shown in Fig. 4 (in this run the growth velocity was $0.8 \mathrm{~nm} / \mathrm{s}$ ). As before the scanning was stopped during the interval I and the tip was positioned in the centre of the triangle under normal tunneling conditions. 


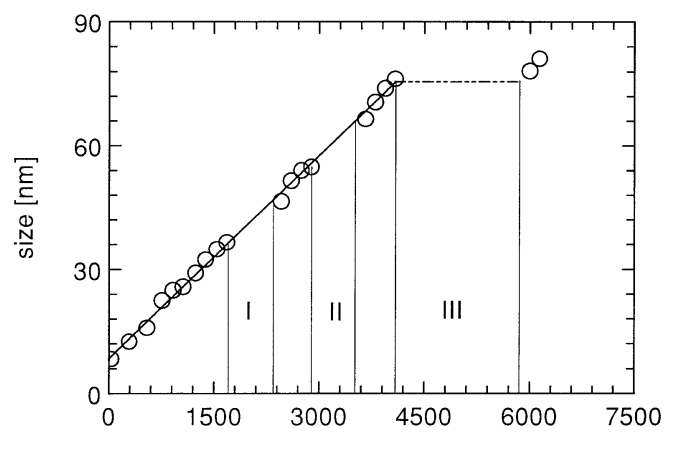

$t[s]$

Fig. 3. Size of a triangular structure as function of time. The scanning was interrupted three times at different sizes of about $35 \mathrm{~nm}$, $55 \mathrm{~nm}$ and $75 \mathrm{~nm}$ respectively: During the intervals I and II the tip was positioned in the center of the scanned area under usual tunneling conditions. During interval III the tip was retracted. Open circles: experimental data; solid line: linear fit

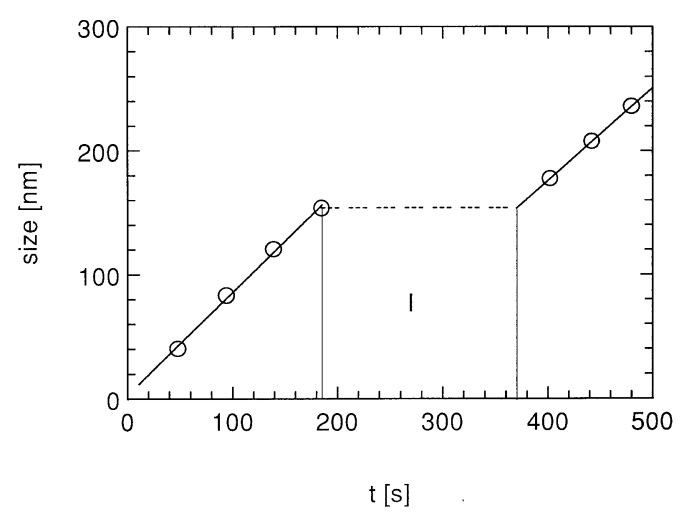

Fig. 4. Size of another triangular structure as function of time with the same conditions as in Fig. 3. This time the scanning was interrupted once (interval I) as the triangle size reached about $160 \mathrm{~nm}$. Open circles: experimental data; solid line: linear fit

In contrast to Fig. 3 no further growth was observed in this case. It is important to note, however, that the side length of the triangle at that point had already reached $160 \mathrm{~nm}$, distinctly larger than in Fig. 3. This difference will be discussed below.

For a further test of the conditions necessary for growth we changed the tunneling voltage from $+1 \mathrm{~V}$ to $-2 \mathrm{~V}$ after some scans (Fig. 5, intervals I and II). As before the tip was not scanned during this process. Apparently the growth is completely suppressed during the tunneling with the opposite bias voltage.

\section{Chemical requirements for the growth}

In order to check for the influence of possible adsorbates, in particular water, on the growth we transferred the $\mathrm{WSe}_{2}$ sample into an $\mathrm{HV}$ chamber. After reaching a pressure of $3 \cdot 10^{-6}$ mbar the same procedure as in air was started. While the same small depression on the sample surface was generated by the voltage pulse, no growth of this structure could be observed within several hours.

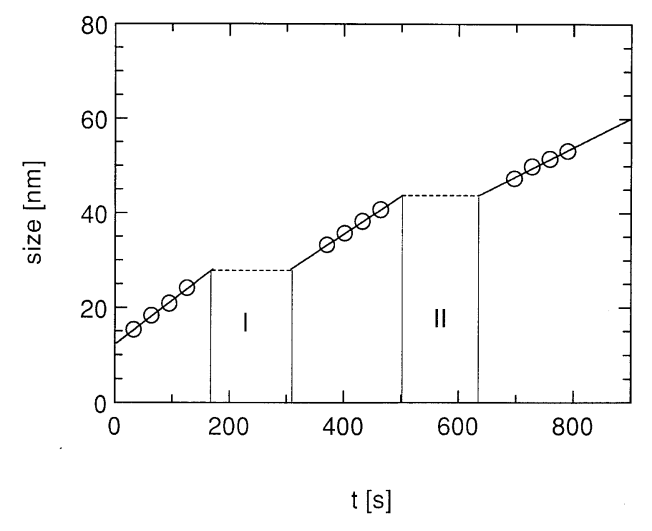

Fig. 5. Size of a triangular structure as function of time, with the scanning being interrupted and the tunneling voltage being changed to $-1 \mathrm{~V}$ during the intervals I and II. Open circles: experimental data; solid line: linear fit

Likewise, no growth was found after venting the HVchamber with dry $\mathrm{N}_{2}$ or dry $\mathrm{O}_{2}$. Only after the $\mathrm{HV}$ chamber was vented with ambient air the triangles were observed to grow again.

These observations suggest a thin water film on the surface of the samples to be essential for the growth of the triangles. In order to confirm this assumption we made an experiment where the whole sample was covered by a droplet of water. For this purpose we first coated the tunneling tip by dipping it into epoxy in order to isolate its surface with the exception of a small area at the tip apex. Even though it was difficult to obtain stable tunneling conditions the growth of triangles could be observed under these conditions as well.

From the experiments in the HV-chamber and the water droplet we conclude that the etching process is directly connected with a physisorbed water layer on the $\mathrm{WSe}_{2}$. Thus we have a situation (tip-water- $\mathrm{WSe}_{2}$ ) similar to experiments in electrochemical cells. By analogy we propose a reaction at the $\mathrm{WSe}_{2}$ surface which is well known from the photochemistry of semiconductors. In electrochemical measurements [7] it was found that carriers and particularly holes $\left(\mathrm{h}^{+}\right)$can cause corrosion of $\mathrm{WSe}_{2}$ according to:

$\mathrm{WSe}_{2}+9 \mathrm{H}_{2} \mathrm{O}+14 \mathrm{~h}^{+} \rightarrow \mathrm{WO}_{3}+2 \mathrm{SeO}_{3}^{2-}+18 \mathrm{H}^{+}$

This reaction can be regarded as a two-stage process: First water is oxidized towards free oxygen, which then reacts with the $\mathrm{WSe}_{2}$ towards soluble tungsten trioxide $\left(\mathrm{WO}_{3}\right)$ and selenite ions. Taking both reactions together the $\mathrm{WSe}_{2}$ is oxidized in the presence of a physisorbed water film due to holes which have to be present at the semiconductor surface.

We now want to discuss to which extent the experimental observations can be accounted for by the proposed model. Obviously the reaction (1) can take place in a water droplet, but neither under vacuum conditions, where the physisorbed water film desorbs, nor in the presence of dry oxygen or nitrogen. The observed large variation of the etching velocities in ambient air may be directly related to the thickness of the physisorbed water layer, which depends on the relative humidity. Moreover a dependence of 
the etching velocity on the $\mathrm{pH}$ of the water has been observed in photochemical reactions [8] as well as a dependence on the partial pressure of gases like $\mathrm{O}_{2}$ in the air [9].

The corrosion reaction is known to run preferentially on non van-der-Waals surfaces [10]. Therefore a defect on the van-der-Waals surface is needed as a nucleus where the reaction can start. This defect is created by the voltage pulse at the beginning of the experiment. On the other hand holes $\left(\mathrm{h}^{+}\right)$are necessary to promote the corrosion reaction. These holes are accumulated below the tip at negative tip bias only, whereas at positive tip bias holes are repelled by the electric field and no reaction can occur (as seen in the intervals I and II of Fig. 5). As the tip has a characteristic curvature holes are accumulated in a defined region below its apex. The experimental data from the experiment with the tip positioned in the center of the triangle show that this region has an extension between about $60 \mathrm{~nm}$ (the triangle is growing in the intervals I and II of Fig. 3) and $160 \mathrm{~nm}$ (no further growth in interval I of Fig. 4) for the tip used here. It seems reasonable that the curvature of the mechanically cut Pt-Ir tips used here is greater than the curvature of the etched Au tips of Enss et al. [5]. Therefore it is clear that a difference is observed in the maximum size that a triangle reaches when the tip is positioned in the center of the triangle without scanning of the tip.

In principle there exists another possible explanation for the experimental results: A chemical reaction at non van-der-Waals surfaces might be triggered by hot electrons, which are injected by the tip at positive sample bias. If this mechanism would hold, one should expect the growth velocity to depend on the magnitude of the tunneling current. Since such a dependence is not observed, as demonstrated by the experiments of Enss et al. [5], this explanation can be ruled out.

Although the experimental observations and the proposed model so far appear to agree very well, an open question exists with respect to results of Parkinson [2], where etching was observed for both positive and negative bias. By contrast we did not find any etching for negative sample bias up to $2 \mathrm{~V}$; at higher bias the surface was damaged in an uncontrollable manner.

In conclusion the etching of $\mathrm{WSe}_{2}$ with an STM on a nanometer scale appears to be governed by the corrosion mechanism (equ. 1) proposed in electrochemistry. It seems worthwhile to complement the investigations described here with an atomic force microscope (AFM), where the necessary bias for growth can be regulated independently from the imaging process. In fact, preliminary AFM observations in our laboratory tend to support the interpretation presented here [11]. On the basis of these results it is possible to write information on a nanometer scale.

We want to thank K. Dransfeld, S. Akari, G. Weiss and C. Enss for stimulating discussions and B. Parkinson for valuable additional information about his measurements. This work was supported by the Deutsche Forschungsgemeinschaft, SFB 306.

\section{References}

1. Quate, C.F.: In: Highlights in Condensed Matter Physics and Future Prospects. Esaki, L. (ed) New York: Plenum Press 1991

2. Parkinson, B.: J. Am. Chem. Soc. 112, 7498 (1990)

3. Akari, S., Möller, R., Dransfeld, K.: Appl. Phys. Lett. 59, (2) 243 (1991)

4. Delawski, E., Parkinson, B.: J. Am. Chem. Soc. 114, 1661 (1992)

5. Enss, C., Winters, R., Reinermann, M., Weiss, G., Hunklinger, S.: (preceeding paper of the same issue)

6. Al-Hilly, A., Evans, B.: J. Cryst. Growth 15, 93 (1972)

7. Lévy-Clément, C., Tenne, R.: In: Photoelectrochemistry and Photovoltaic of Layered Semiconductors, A. Aruchamy, (ed) London, New York: Kluwer 1992

8. Sinn, C.: Dissertation, University of Hamburg 1989

9. Mahalu, D., Peisach, M., Jaegermann, W., Wold, A., Tenne, R.: J. Phys. Chem. 94, 8012 (1990)

10. Kautek, W., Gerischer, H.: Surf. Sci. 119, 60 (1982)

11. Böhmisch, M., Burmeister, F., Boneberg, J., Lux-Steiner, M., Leiderer, P.: (to be published) 